\title{
Ion pair chromatograpy: a critical prespective
}

\begin{abstract}
Reversed phase chromatography is one of most popular modes of chromatography in use. Ion pair chromatography a subset of reverse phase chromatography even though happens due to ionic interactions, needs a reverse phase stationary phase as support. Retention of basic analytes on reverse phase columns is the most commonly encountered situation where ion pair chromatography is used. Reproducibility problems in gradient elution, slow equilibration times are some of the disadvantages of ion pair chromatography for which mixed mode columns can provide the best possible solution. In this concise review, an attempt has been made to give an overview of all aspects of ion pair chromatography.
\end{abstract}

Keywords: ion pair chromatography, ion-pair agents, octane sulfonic acid, trifluoroacetic acid, mixed mode columns
Volume 4 Issue 6 - 2017

\section{Charu K Yerneni}

Department of Analytical R\&D, Purdue University, USA

Correspondence: Charu K Yerneni, Scientist III, Department of Analytical R\&D, Purdue University, Tris Pharma Inc., Suite D, Route 130 S, Monmouth Junction, New Jersey, USA, Fax 173286I2726, Email cyerneni@trispharma.com

Received: April 27, 2017 | Published: May 15, 2017

\section{Introduction}

There is no exaggeration in saying that High Performance Liquid Chromatography has helped the pharmaceutical industry to grow by leaps and bounds. ${ }^{1}$ Most workers using reverse phase chromatography prefer silica based columns in the low $\mathrm{pH}$ range. Silica based columns available today are very robust in the $\mathrm{pH}$ range 2 to 8 . Since most of the reversed phase chromatography is run in the $\mathrm{pH}$ between 2 to 3 , these conditions are best suitable for the retention of acidic molecules. Neutral molecules would show significant retention irrespective of $\mathrm{pH}$. But the issue is with the basic compounds which cannot be retained on the column successfully because they remain ionized at acidic $\mathrm{pH}$. In cases where samples have both acids and bases ion pairing might be a best option.

\section{What is ion pairing?}

Reverse phase separation is a result of interaction between non polar molecules and non-polar stationary phase. Retention can be adjusted by varying the organic percentage of the mobile phase. Most non polar molecules and acidic molecules due to low $\mathrm{pH}$ of the mobile phase can be made to retain well on the column for a good quality separation. However basic molecules which remain ionized at low $\mathrm{pH}$ are the ones that are hard to retain. This is where the ion-pair agents ${ }^{2}$ come in handy. Basically ion pair reagents have a polar head and a non-polar tail made of long carbon chains. One example is octane sulfonic acid. The non-polar end gets attracted to non-polar stationary phase leaving its charged end hanging in the mobile phase. The basic species can now get attracted to the oppositely charged immobilized ion pair reagents thus causing chromatographic retention ${ }^{3}$ (Figure1). The pairing may also happen in the bulk of the mobile phase which gives rise to a neutral molecule that interacts with the non-polar stationary phase. So a negatively charged ion pair reagent can be used to retain positively charged basic molecules.

\section{Downsides of ion pair chromatography}

Gradient elution is a difficult task in ion pair chromatography. Slow equilibration between mobile phase and column is another issue. For example a $150 \mathrm{x} 4.6 \mathrm{~mm}$ column might need up to $1 \mathrm{mmol}$ of ion pair reagent for complete coverage by an ion pair agent. At a concentration of $5 \mathrm{mM}$, it may take at least $200 \mathrm{ml}$ to load up the column. At a flow rate of $1 \mathrm{ml} / \mathrm{min}$ it may take up to 4 hours for equilibration. Also ion pair reagents due to strong hydrophobic interactions cannot be completely flushed from the column even after extensive washing. This means that dedicating a column to a particular ion pair application is very important. Since ion pair reagents show significant absorbance in the lower UV region, method development in lower UV wavelength region might be a difficult task due to interferences in absorbance from the mobile phase components.

\section{Alternative approaches}

Some of the alternative approaches to ion pair reagents are using

\section{a. Trifluoroacetic $\operatorname{acid}^{5}$}

b. Mixed mode columns.

Trifluoroacetic acid is a commonly used ion pair reagent nowadays for biological samples like proteins and peptides. ${ }^{6}$ Trifluoroacetic acid also equilibrates faster and can be used with gradient elution.

Today's chromatography market is flooded with different kinds of mixed mode columns ${ }^{7}$ which are a convenient alternative to ion pair chromatography. For example polar embedded groups ${ }^{8}$ have a positive or negative charge at the base of carbon chain which provide both ionic and reverse phase character and can possibly eliminate the need of an ion pair agent.

\section{Conclusion}

Even though ion pair chromatography has its shortcomings in some cases it might give you the best possible solution. Make sure to give enough time for equilibration when using ion pair reagent based mobile phases. Try to explore other options like using trifluoroacetic acid, mixed mode columns etc.

\section{Acknowledgments}

None.

\section{Conflicts of Interset}

None.

\section{References}

1. Nikolin B, Imamović B, Medanhodzić VS, et al. High perfomance liquid chromatography in pharmaceutical analyses. Bosn J Basic Med Sci. 2004;4(2):5-9.

2. Fallon A, Booth RFG, Bell LD. Chapter 8 High performance reverse phase ion-pair chromatography. Laboratory Techniques in Biochemistry and Molecular Biology. 1987;17:90-101. 
3. Dolan JW. Ion Pairing - Blessing or Curse? LCGC. 2008;21(5).

4. Zhang J, Raglione T, Wang Q, et al. Regeneration of tetrabutylammonium ion-pairing reagent distribution in a gradient elution of reversed phase ion-pair chromatography. J Chromatogr Sci. 2011;49(10):825-831.

5. Cai B, Li J. Evaluation of trifluoroacetic acid as an ion-pair reagent in the separation of small ionizable molecules by reversed-phase liquid chromatography. Analytica Chimica Acta. 1999;399(3):249-258.

6. Shibue M, Mant CT, Hodges RS. Effect of anionic ion-pairing reagent hydrophobicity on selectivity of peptide separations by reversed-phase liquid chromatography. J Chromatogr A. 2005;1080(1):68-75.
7. Zhang K, Liu X. Mixed-mode chromatography in pharmaceutical and biopharmaceutical applications. J Pharm Biomed Anal. 2016;128:7388 .

8. Wilson NS, Gilroy J, Dolan JW, et al. Column selectivity in reversedphase liquid chromatography: VI. Columns with embedded or endcapping polar groups. J Chromatogr A. 2014;1026(1-2):91-100. 